\section{Acute encephalopathy with biphasic seizures and late reduced diffusion associated with Streptococcus sanguinis sepsis}

\author{
Hitoshi Awaguni, Jun Shinozuka, \\ Shin-ichiro Tanaka, Sayaka Kadowaki, \\ Shigeru Makino, Rikken Maruyama, \\ Yosuke Shigematsu, Kenji Hamaoka, \\ Shinsaku Imashuku \\ Division of Pediatrics, Uji-Tokushukai \\ Medical Center, Uji, Kyoto, Japan
}

\begin{abstract}
Acute encephalopathy with biphasic seizures and late reduced diffusion (AESD) develops in association with systemic as well as central nervous system (CNS) viral or bacterial infections. AESD is most often noted with influenza or human herpesvirus 6 infection in previously healthy infants. However, AESD has also been reported in an infant with developmental retardation and in a mentally and motor-disabled adolescent. Here, we report the case of a 4year-old female with significant development delay due to spinal muscular atrophy, who developed AESD during Streptococcus sanguinis sepsis with no apparent CNS infection. Although the patient had extremely high serum procalcitonin $(45.84 \mathrm{ng} / \mathrm{mL}$, reference; $<0.4$ ) on admission indicating a poor prognosis, she was successfully managed for sepsis and AESD.
\end{abstract}

\section{Introduction}

Acute encephalopathy with biphasic seizures and late reduced diffusion (AESD) is a rare encephalopathy that is mostly reported in Japan. ${ }^{1}$ AESD was originally described as the presentation of clinical biphasic seizures on day 1 and days 4-6 accompanied by radiological findings showing no or mild acute abnormality on days $1-2$, followed by magnetic resonance imaging (MRI) findings showing reduced diffusion in subcortical white matter on days 3-9.2 AESD mainly occurs in previously healthy infants, and is associated with viral infections such as influenza ${ }^{2}$ or human herpesvirus 6 (HHV-6), ${ }^{3}$ as well as bacterial infections. ${ }^{4-6}$ AESD has also been reported in an infant with developmental retardation 4 and in a mentally and motor-disabled adolescent. ${ }^{7}$ Here, we report a case of AESD caused by bacterial infection (Streptococcus sanguinis $[S$. sanguinis $]$ sepsis, but not meningitis) in a 4-year-old female with significant developmental delay.

\section{Case Report}

The patient was born full term with a birth weight of $3,184 \mathrm{~g}$, and was the secondborn child of her family. She was suspected to have developmental delay at the age of 6 months and was diagnosed with spinal muscular atrophy (SMA) ${ }^{8}$ at the age of 1 year. It was not determined if she had acute infantile SMA (type I) or chronic infantile SMA (type II). ${ }^{9}$ She became bed-ridden, could not roll over, and had a weak cough reflex. She had been able to take food with help, but needed biphasic positive airway pressure (BiPAP) apparatus to overcome sleep apnea syndrome until at the age of 4 years and 3 months, when she developed infection-related hemophagocytic lymphohistiocytosis (HLH; for which she fulfilled 5/8 of the clinical diagnostic criteria) ${ }^{10}$ with significantly abnormal laboratory data (serum brain natriuretic peptide $>2,000 \mathrm{pg} / \mathrm{mL}$; aspartate aminotransferase $>13,000 \mathrm{U} / \mathrm{L}$; lactate dehydrogenase $>10,000 \mathrm{U} / \mathrm{L}$; and ferritin, $7590 \mathrm{ng} / \mathrm{mL}$ ), and suffered from severe dilated cardiomyopathy (Ejection Fraction, 30\%; Mitral Regurgitation grade III). Fortunately, the patient survived this episode with methylprednisolone pulse therapy and hemodynamic and respiratory support. Eventually, tracheotomy and gastrostomy were performed and she was able to receive home care after discharge.

At the age of 4 years and 10 months, she was transferred to the emergency clinic with loss of consciousness associated with severe hypoglycemia (blood glucose, 10 $\mathrm{mg} / \mathrm{dL}$ ). On day 1 of admission, her physical condition was estimated as Japan Coma Scale (JCS) III-200 with blood pressure, 110/62 $\mathrm{mmHg}$; heart rate, 106/min; respiration rate, 30/min; and $\mathrm{SpO} 2,90 \%$ (room air). Along with persistent disturbed consciousness, the patient showed prolonged episodes of facial spasms and one-point stare-type seizures, associated with abnormal electroencephalogram (EEG), which revealed diffuse high-voltage slow wave pattern. No generalized seizures were noted. Her laboratory data were as follows: white blood cell count, 30,000/ $\mu \mathrm{L}$ (neutrophils, 89\%); Hb, $14.0 \mathrm{~g} / \mathrm{dL}$; platelet count, $421 \mathrm{~K} / \mu \mathrm{L}$; serum alanine aminotransferase, $19 \mathrm{U} / \mathrm{L}$; lactate dehydrogenase, $360 \mathrm{U} / \mathrm{L} ; \mathrm{BUN}, 33 \mathrm{mg} / \mathrm{dL}$; creatinine 0.16 $\mathrm{mg} / \mathrm{dL}$; blood glucose, $10 \mathrm{mg} / \mathrm{dL}$; C-reactive protein (CRP), $0.13 \mathrm{mg} / \mathrm{dL}$; procalcitonin, 45.84 (reference $<0.4$ ) $\mathrm{ng} / \mathrm{mL}$; and
Correspondence: Shinsaku Imashuku, Division of Pediatrics, Uji-Tokushukai Medical Center, Uji, Kyoto 611-0041, Japan.

Tel.: +81.774.20.1111 - Fax: +81.774.20.2336.

E-mail: shinim95@mbox.kyoto-inet.or.jp

Key words: Acute encephalopathy with biphasic seizures and reduced diffusion, AESD, spinal muscular atrophy, Streptococcus sanguinis, sepsis.

Acknowledgments: the authors thank all staff members who took care of the patient, particularly Dr. Atsushi Yokoyama, Kyoto University, for his advices.

Contributions: the authors contributed equally.

Conflicts of interest: the authors declare no potential conflict of interest.

Funding: none.

Received for publication: 27 September 2017.

Revision received: 22 Jnauary 2018.

Accepted for publication: 12 February 2018.

This work is licensed under a Creative Commons Attribution NonCommercial 4.0 License (CC BY-NC 4.0).

(C) Copyright H. Awaguni, et al., 2018

Licensee PAGEPress, Italy

Pediatric Reports 2018; 10:7424

doi:10.4081/pr.2018.7424

serum ferritin, $85 \mathrm{ng} / \mathrm{mL}$. Blood gas showed a base excess of $-8.2 \mathrm{mmol} / \mathrm{L}$. After glucose infusion, cerebrospinal fluid (CSF) was examined, which showed cell counts of $1 / \mu \mathrm{L}$; protein, $14 \mathrm{mg} / \mathrm{dL}$; and glucose, 60 $\mathrm{mg} / \mathrm{dL}$. Tests for viral infections such as influenza A/B and HHV-6 were negative. Blood culture revealed the presence of $S$. sanguinis, thus, the patient was diagnosed as having $S$. sanguinis sepsis; however, clear CSF findings meant that she had no CNS bacterial infections. She was immediately treated with ceftriaxone, midazolam, and fosphenytoin sodium hydrate, and, from days 2 to 5 , methylprednisolone pulse therapy. The above measures were effective to control sepsis, to obtain spasmolysis and consciousness recovery. However, the patient lost consciousness again on day 5 in association with similar episodes of facial spasms and one-point stare seizures. This time, intravenous immunoglobulin (2 g) together with midazolam and diazepam was administered. Dextromethorphan was also given considering its efficacy for encephalopathy. ${ }^{11}$ The first brain MRI (diffusion weighted imaging, DWI) taken on day 2 of admission showed mild localized 
high signal intensities in the right temporal lobe and left parietal lobe (Figure 1A). However, DWI on day 5 showed bright-tree like features of bilateral generalized high signal intensities in the cerebral hemispheres, which was suggestive of severe acute encephalitis/encephalopathy in a biphasic pattern (Figure 1B). These clinical and radiological findings were compatible with the diagnosis of AESD. Although follow-up brain DWI on day 16 still showed high signal intensities bilaterally in the cerebral hemispheres, the patient gradually recovered with normal EEG findings and discharged.

\section{Discussion}

We report the case of AESD in a disabled child with SMA, which is a motor neuron disease caused by SMN1 gene mutation. Infants with type 1 SMA, the most severe form, usually die within months or a few years due to respiratory insufficiency and bulbar paralysis. ${ }^{8}$ Our patient, who had respiratory problems, has survived beyond the age of 4 years old; however, during her infancy, she developed severe dilated cardiomyopathy at the time of HLH episode. Since no clear correlation has been confirmed between SMA and cardiac involvement, 9 her cardiomyopathy could indicate one of HLH-related organ failures. 12 AESD developed in this SMA patient, 7 months after the episode of HLH.

Patients with AESD initially show a prolonged febrile seizure, which is followed by subsequent seizure occurring after several days of interval. Although the initial neu-

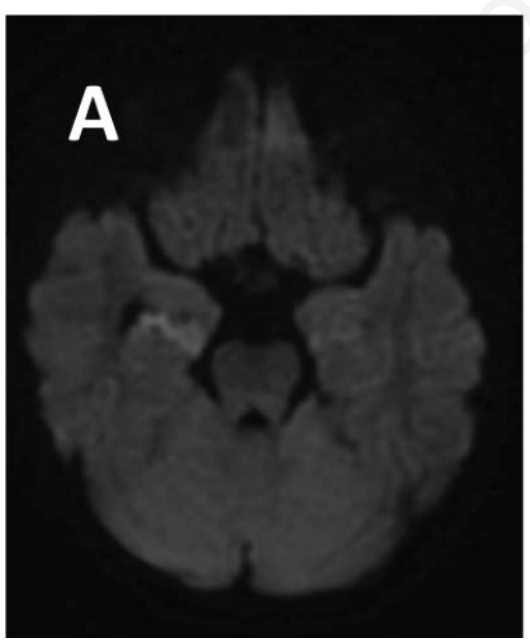

Figure 1. Magnetic resonance imaging diffusion weighted imaging on day 2 showed a localized high signal intensity in the right temporal lobe (a left parietal lobe lesion is not shown on this figure) (A) and, on day 5 showed generalized high signal intensities in the cerebral hemispheres bilaterally (B), indicating a biphasic pattern.

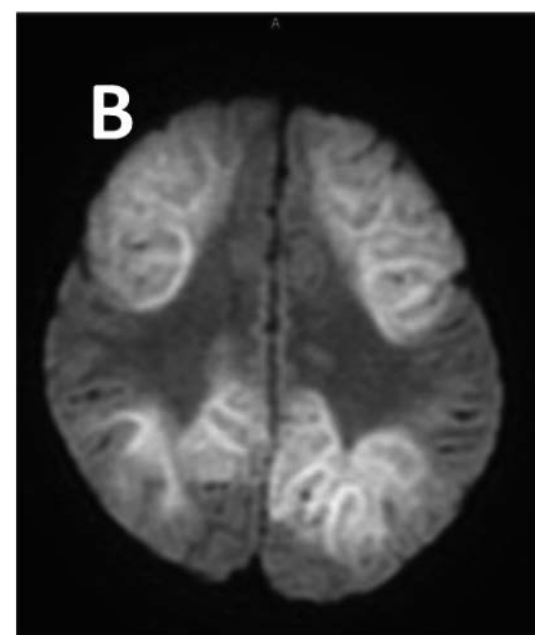

rologic symptoms are typically generalized seizures, but other symptoms such as nonconvulsive status epilepticus, ${ }^{6}$ or involuntary movements are also described. ${ }^{13}$ It remains unknown if the partial seizures such as facial spams/one- point stare in our case were related to SMA, in which myoclonic seizures have been reported. ${ }^{14}$ On MRI, she showed only mild DWI changes at day 2 , and at day 5 typical bright-tree like characteristic DWI images. Thus, her clinical course of AESD was typical as described previously.1,2 In an analysis of 62 cases of AESD, 1 Tada et al. proposed an AESD prediction score system based on initial symptoms/laboratory data differentiating AESD from prolonged febrile seizures (mean scores 5.9 vs. 1.8 out of 9). In fact, our patient had score 4 out of 8 , because mechanical ventilation was already placed for SMA.

S. sanguinis is a Gram-positive coccus and a member of the Viridans Streptococcus group. Although $S$. sanguinis is a normal inhabitant of the mouth in healthy humans, it may cause subacute bacterial endocarditis in patients even without structurally abnormal heart valves. 15 In pediatrics, viridans streptococcus (including $S$. sanguinis) sepsis was mostly described in patients with cancer receiving chemotherapy. ${ }^{16}$ Our patient was not immuno-compromised, was not at risk of implantable venous port-associated bloodstream infections, and had not received specific procedures of recent dental cleaning or surgery. It is possible that her SMA-related respiratory dysfunction and HLH-induced cardiac disease became risk factors for $S$. sanguinis-induced sepsis.

Our patient developed AESD in associthat procalcitonin is a better marker of sepsis than CRP. ${ }^{19}$ In terms of diagnostic and prognostic value, serum procalcitonin on day 1 has been shown to be significantly higher in patients with than without septic shock [median, 14 (0.3-767) ng/mL vs. 1 (0.5-36) ng/mL, $\mathrm{P}<0.01] .{ }^{20}$ In addition, a cutoff value of $6 \mathrm{ng} / \mathrm{mL}$ on day 1 separated patients who died from those who survived with $87.5 \%$ sensitivity and $45 \%$ specificity. 20 Thus, in patients with sepsis or septic shock, procalcitonin becomes a prognostic marker. 20,21 Fortunately, with intensive treatments, procalcitonin of our patient declined to $0.38 \mathrm{ng} / \mathrm{mL}$ at day 8 , and she survived regardless of significantly high serum levels on admission.

\section{Conclusions}

This paper describes a unique case of AESD; the patient developed S. sanguinis sepsis-related AESD with underlying SMA, 
and fully recovered. However, since the eventual outcome of AESD is thought not to be bright, ${ }^{1}$ we plan to carry out long-term follow-up of mental and physical development in this patient.

\section{References}

1. Tada H, Takanashi J, Okuno H, et al. Predictive score for early diagnosis of acute encephalopathy with biphasic seizures and late reduced diffusion (AESD). J Neurol Sci 2015; 358:62-5.

2. Takanashi J, Tsuji M, Amemiya K, et al. Mild influenza encephalopathy with biphasic seizures and late reduced diffusion. J Neurol Sci 2007;256:86-9.

3. Kawamura Y, Yamazaki Y, Ohashi M, et al. Cytokine and chemokine responses in the blood and cerebrospinal fluid of patients with human herpesvirus 6Bassociated acute encephalopathy with biphasic seizures and late reduced diffusion. J Med Virol 2014;86:512-8.

4. Kuwata S, Senzaki H, Urushibara Y, et al. A case of acute encephalopathy with biphasic seizures and late reduced diffusion associated with Streptococcus pneumoniae meningoencephalitis. Brain Dev 2012;34:529-32.

5. Yokochi T, Sakanishi S, Ishidou Y, et al. Acute encephalopathy with biphasic seizures and late reduced diffusion associated with staphylococcal toxic shock syndrome caused by burns. Brain Dev 2016;38:875-9.

6. Yamaguchi H, Tanaka T, Maruyama A, Nagase H. Septic encephalopathy char- acterized by acute encephalopathy with biphasic seizures and late reduced diffusion and early nonconvulsive status epilepticus. Case Rep Neurol Med 2016;2016:7528238.

7. Bekci T, Aslan K, Bilgici MC, et al. A missed diagnosis: acute encephalopathy with biphasic seizures and late reduced diffusion. Clin Neurol Neurosurg 2014;127:161-2.

8. Shababi M, Lorson CL, RudnikSchöneborn SS. Spinal muscular atrophy: a motor neuron disorder or a multiorgan disease? J Anat 2014;224:15-28.

9. Palladino A, Passamano L, Taglia A, et al. Cardiac involvement in patients with spinal muscular atrophies. Acta Myol 2011;30:175-8.

10. Henter JI, Horne A, Aricó M, et al. HLH-2004: Diagnostic and therapeutic guidelines for hemophagocytic lymphohistiocytosis. Pediatr Blood Cancer 2007;48:124-31.

11. Matsuo M, Maeda $\mathrm{T}$, Ono $\mathrm{N}$, et al. Efficacy of dextromethorphan and cyclosporine for acute encephalopathy. Pediatr Neurol 2013;48:200-5.

12. Karapinar B, Yilmaz D, Balkan C, et al. An unusual cause of multiple organ dysfunction syndrome in the pediatric intensive care unit: hemophagocytic lymphohistiocytosis. Pediatr Crit Care Med 2009;10:285-90.

13. Lee S, Sanefuji $M$, Torio $M$, et al. Involuntary movements and coma as the prognostic marker for acute encephalopathy with biphasic seizures and late reduced diffusion. J Neurol Sci 2016;370:39-43.
14. Rubboli G, Veggiotti P, Pini A, et al. Spinal muscular atrophy associated with progressive myoclonic epilepsy: A rare condition caused by mutations in ASAH1. Epilepsia 2015;56:692-8.

15. Inada T, Shirono K, Tsuda H. Hemolytic anemia in a patient with subacute bacterial endocarditis due to Streptococcus sanguis. Acta Haematol 1995;94:95-7.

16. Ahmed R, Hassall T, Morland B, Gray J. Viridans streptococcus bacteremia in children on chemotherapy for cancer: an underestimated problem. Pediatr Hematol Oncol 2003;20:439-44.

17. Thi VA, Nordmann P, Landrieu P. [Encephalopathy associated with severe bacterial infections: presuppurative encephalitis or toxi-infectious disorder?]. Rev Neurol (Paris) 2002;158 709-12. [Article in French]

18. Banks J, Poole S, Nair SP, et al. Streptococcus sanguis secretes CD14binding proteins that stimulate cytokine synthesis: a clue to the pathogenesis of infective (bacterial) endocarditis? Microb Pathog 2002;32:105-16.

19. Luzzani A, Polati E, Dorizzi R, et al. Comparison of procalcitonin and $\mathrm{C}$ reactive protein as markers of sepsis. Crit Care Med 2003;31:1737-41.

20. Clec'h C, Ferriere F, Karoubi P, et al. Diagnostic and prognostic value of procalcitonin in patients with septic shock. Crit Care Med 2004;32:1166-9.

21. Carrol ED, Newland P, Thomson AP, Hart CA. Prognostic value of procalcitonin in children with meningococcal sepsis. Crit Care Med 2005;33:224-5. 